\title{
FATHER TIME. II. A PHYSICAL BASIS BEHIND FEYNMAN'S IDEA OF ANTIPARTICLES MOVING BACKWARD IN TIME, AND AN EXTENSION OF THE CPT THEOREM TO INCLUDE NON-LOCAL GAUGE FIELDS
}

\author{
T K Rai Dastidar* and Krishna Rai Dastidar† \\ Indian Association for the Cultivation of Science, Calcutta 700 032, India
}

\begin{abstract}
It was demonstrated in a recent paper (Mod.Phys.Lett. A13, 1265 (1998) ; hepth/9902020) that the existence of a non-thermodynamic arrow of time at the atomic level is a fundamental requirement for conservation of energy in matter-radiation interaction. Since the universe consists of two things only - energy and massive matter - we argue that as a consequence of this earlier result, particles and antiparticles must necessarily move in opposite directions in time. Our result further indicates that the CPT theorem can be extended to cover non-local gauge fields.
\end{abstract}

\footnotetext{
* Atomic \& Molecular Physics Group, Department of Materials Science. Electronic address : mstkrd@mahendra.iacs.res.in

$\dagger$ Department of Spectroscopy. Electronic address : spkrd@mahendra.iacs.res.in
} 


\section{Introduction}

Some time ago [1] we obtained a result that matter fields possess a non-local U(1) gauge transformation symmetry that necessitates invoking a non-local electromagnetic field, and that a non-thermodynamic arrow of time at the quantum (atomic) level is enforced upon us as a necessary condition for conservation of energy in matter-radiation interactions. Based upon this result, it was subsequently predicted [2] that the spectrum of a blackbody radiation passing through gaseous matter would show an apparent deviation from the Planck formula. A very simple experimental test for verifying this prediction has been suggested in [2], where it was also shown that the results of a balloon-borne measurement [3] of the cosmic microwave background radiation (CMBR) spectrum does seem to show such a feature ${ }^{\mathrm{a}}$.

Subject to verification of the above theoretical prediction, we argued recently [5] that, as a necessary condition for energy conservation in matter-radiation interactions, all particles in the universe subject to electromagnetic interactions should follow this one and the same arrow of time through the agency of the CMBR ${ }^{\mathrm{b}}$. The present paper, which is a companion to [5], addresses reactions which do not conserve energy, but involve mass $\leftrightarrow$ energy conversion, e.g. particle-antiparticle annihilation, pair production, particle decay etc.

\section{Theory and Results}

To make the present paper self-contained, we briefly recapitulate here how the time's arrow appears in the theory as a necessary condition for conservation of energy in the atomic level. (See [1] for details.) A matrix element for matter-radiation interaction with the non-local electromagnetic field potential $\mathcal{A}\left(x, x^{\prime}\right)$ where $x \equiv(\mathbf{r}, t)$ is typically of the form

$$
M_{f i}(t) \propto\left\langle\psi_{f}(x), n_{f}\left|\hat{p} \cdot \mathcal{A}\left(x, x^{\prime}\right)\right| \psi_{i}\left(x^{\prime}\right), n_{i}\right\rangle
$$

the integration running over $d^{3} r$ and $d^{4} x^{\prime}$. Special relativity requires that the time integral over $d t^{\prime}$ should run from $-\infty$ to $(t-\rho / c)$ [where $\left.\rho=\left|\mathbf{r}-\mathbf{r}^{\prime}\right|\right]$ for the retarded interaction, and from $(t+\rho / c)$ to $+\infty$ for the advanced interaction. After Fourier expanding $\mathcal{A}$ in two sets of photon modes (see, e.g. [6]), the time integration over $d t$ gives us the energyconserving delta-function $\delta\left(E_{f}, E_{i}+\hbar \omega+\hbar \omega^{\prime}\right)$ in the correlated two-photon absorption process (see below; this is one of the new results in [1]). Formally, this time integration runs from $t=-\infty$ to $t=+\infty$. However, if we agree to define that the atom has been excited, i.e. the matrix element has come into existence, at the time $t=0$, then it is

${ }^{\text {a }}$ In [2] we also discuss why the cosmic background explorer $(C O B E)$ satellite measurements of the CMBR spectrum [4] do not show this feature.

$\mathrm{b}$ The restriction, that the particles must be subject to electromagnetic interactions, simply reflects the present status of the theory (which is undergoing continual development), and there seems to be no reason why it should be fundamental ; work is in progress to see if it can be removed. 
at once obvious that the segment $\int_{-\infty}^{0} d t$ does not contribute anything to the $t$-integral, giving finally a double integral to be chosen from

$$
\int_{0}^{\infty} f(t) d t\left(\int_{-\infty}^{t-\rho / c} g\left(t^{\prime}\right) d t^{\prime}, \quad \int_{t+\rho / c}^{\infty} g\left(t^{\prime}\right) d t^{\prime}\right)
$$

where $f(t)=\exp \left[\frac{i}{\hbar}\left(E_{f} t-\hbar \omega t\right)\right], g\left(t^{\prime}\right)=\exp \left[-\frac{i}{\hbar}\left(E_{i} t^{\prime}+\hbar \omega^{\prime} t^{\prime}\right)\right]$. It is obvious that the retarded interaction gives the required $\delta$-function for energy conservation, while the advanced interaction fails to do so. Thus we are constrained to limit the non-local temporal correlation to the past only ; the fundamental principle of energy conservation has given us an arrow of time, i.e. the principle of causality, as a corollary. ${ }^{c}$

We are thus led to the result that in atomic-scale reactions, forward motion in time of all the reactants/products is a necessary condition for conservation of energy. What happens if energy is not conserved ? The universe contains but two things - energy and massive matter - and mass $\leftrightarrow$ energy conversion is the only possible outcome of such reactions. However, according to our earlier result, such "non-conservation" of energy in atomic-scale reactions can take place if and only if one or more of the reactants/products do not move forward in time, i.e. move backward in time (since time is one-dimensional).

We may ask the question from the opposite viewpoint : what happens in a reaction if one or more of the reactants/products travel backward in time ? According to [1], the answer is simple ; energy will not be conserved, hence mass $\leftrightarrow$ energy conversion must occur in such reactions. (Of course, the precise time interval between the "start" of the reaction and the mass $\leftrightarrow$ energy conversion depends on the particular characteristics in the process involved ; for example, positron-electron annihilation can take place either instantaneously or after positronium formation, depending on the collision geometry.)

We see therefore that from our time's arrow, we have obtained the result that in reactions where mass $\leftrightarrow$ energy conversion takes place, at least one of the reactant/product particles must be travelling in time in a direction opposite to the others. When we recall that such reactions must involve both "particles" and "antiparticles", then from known properties of antiparticles, we conclude that an antiparticle can be obtained only under a combined operation of charge conjugation $\mathrm{C}$, parity $\mathrm{P}$ and time reversal $\mathrm{T}$ (in any order) on a particle ; similarly, CPT operating on an antiparticle gives us a particle. Since the total number of particles and antiparticles in any process involving particles and antiparticles is always even, the matrix element for the process remains invariant under the CPT operation. (This invariance is not quite self-evident, as we remember that the CPT theorem is supposed to be valid for local field theories only ; our work seems to have broadened its scope.)

c As shown in [1], however, we have to pay a price for it ; the correlation with the "past" is to be understood in an EPR-like sense and not in a Lorentz invariant sense, if we are to maintain energy conservation. (This serves to remind us that quantum mechanics and special relativity have always been strange bedfellows.) It is, of course, just a convention of language that we visualise particles, i.e. the constituents of our own universe as moving "forward" in time, thus establishing a causal link with the "past" and not with the "future". 
To conclude, we have found a physical basis for Feynman's idea that antiparticles can be considered as particles moving backward in time, and we have also found that the scope of the CPT theorem can be extended to cover non-local gauge fields.

We thank Dr J Chakravarti, Department of Theoretical Physics for his interest in the ongoing work. 


\section{References}

1. T K Rai Dastidar and Krishna Rai Dastidar, Mod. Phys. Lett. A13, 1265 (1998) ; Errata A13, 2247 (1998) ; hep-th/9902020

2. T K Rai Dastidar, Mod. Phys. Lett. A 14, 1193 (1999) ; quant-ph/9903043

3. D P Woody and P L Richards, Phys. Rev. Lett. 42, 925 (1979)

4. J C Mather et al, Astrophys. J. 420, 439 (1994)

5. T K Rai Dastidar, "Father Time. I. Does the Cosmic Microwave Background Radiation Provide a Universal Arrow of Time?" quant-ph/9903053 (To appear in Mod. Phys. Lett. A).

6. I M Gel'fand and G E Shilov, Generalised Functions (Acad. Press, 1964) Vol. 1, Chap. 2, eq. 1.3(1) 